\title{
Le malentendu comme structure de la communication
}

Communication as Patterned by Misunderstanding

\section{Christine Servais et Véronique Servais}

\section{(2) OpenEdition}

12 Journals

Édition électronique

URL : http://journals.openedition.org/questionsdecommunication/432

DOI : 10.4000/questionsdecommunication.432

ISSN : 2259-8901

\section{Éditeur}

Presses universitaires de Lorraine

\section{Édition imprimée}

Date de publication : 1 juillet 2009

Pagination : 21-49

ISBN : 978-2-86480-989-0

ISSN : 1633-5961

\section{Référence électronique}

Christine Servais et Véronique Servais, "Le malentendu comme structure de la communication », Questions de communication [En ligne], 15 | 2009, mis en ligne le 01 août 2011, consulté le 30 avril 2019. URL : http://journals.openedition.org/questionsdecommunication/432 ; DOI : 10.4000/ questionsdecommunication.432 


\title{
$>$ DOSSIER
}

CHRISTINE SERVAIS

Médiation esthétique et communication

Université de Liège

Christine.Servais@ulg.ac.be

VÉRONIQUE SERVAIS

Laboratoire d'anthropologie sociale et culturelle

Université de Liège

v.servais@ulg.ac.be

\section{LE MALENTENDU COMME STRUCTURE DE LA COMMUNICATION}

\begin{abstract}
Résumé. - Dans les modèles télégraphiques, on considère que la communication fonctionne si les messages de départ et d'arrivée sont identiques. Sinon, cela signifie que le récepteur a mal compris ou que l'émetteur s'est mal fait comprendre. Dans ce cadre, la pathologie de la communication est l'incompréhension. Ce que nous proposons ici, à partir de l'analyse de situations de communication très particulières (des rencontres enchantées avec des dauphins sauvages), c'est que, à l'inverse, la structure fondamentale de la communication est le malentendu. Or, c'est le refus de reconnaître ce fait (dû notamment au désir vain et désastreux de contrôler le partenaire) qui engendre des pathologies. Admettre au contraire qu'émetteur et récepteur disposent nécessairement de versions différentes de l'interaction et qu'il n'y a pas de version plus objective qu'une autre, c'est replacer l'altérité au cœur d'une communication qui est réussie parce qu'on accepte de mal se comprendre.
\end{abstract}

Mots clés. - Malentendu, communication inter-espèces, interaction, dauphins, différend, mésentente. 
S i la communication peut être considérée comme pathologique c'est-à-dire provoquant des désordres, des souffrances ou des aberrations quand elle fonctionne trop bien - peut-on, à l'inverse, considérer que, pour éviter qu'elle n'engendre des pathologies, il faut admettre qu'elle repose sur un malentendu ? C'est la thèse que nous défendons dans ce texte. Avant nous, d'autres ont avancé l'idée que « la compréhension [était] un cas particulier du malentendu »' (Culioli, 1990 : 39). Toutefois, cette formule ne remet pas en question le présupposé selon lequel une bonne communication repose sur la transparence de l'intercompréhension : elle suggère juste que cela survient rarement. Le fait est que les recherches en information et communication sont encore aujourd'hui confrontées à des difficultés qui les travaillent depuis leur émergence et qui sont relatives à une tension entre le champ du savoir et celui de l'action. En effet, dès qu'il y eût conscience de l'importance des activités de communication dans les sociétés, celles-ci furent rapportées à des enjeux qui déterminèrent, pour partie, la pertinence des modèles. La portée empirique des processus de communication a directement conduit à ce que médias et techniques soient pris dans une visée d'instrumentalisation : les médias de masse, relais de l'émancipation des masses ou, au contraire, de leur abrutissement ; les techniques de communication, relais de tous les désirs de manipulations, et actuellement, la culture, relais d'une resocialisation, d'un remaillage du tissu social par le biais de la médiation culturelle... Toutes ces conceptions reposent sur l'idée qu'il existe une bonne et une mauvaise communication : la bonne étant une communication réussie, c'est-à-dire débouchant sur des effets conformes à l'intention de l'émetteur ; la mauvaise échouant à les faire coïncider. Une communication efficace est donc considérée comme transmission et non comme véritable médiation, qui, quant à elle, suppose un rapport plus compliqué à l'altérité. Qu'on la pratique ou qu'on s'en offusque, la communication est un quasi-synonyme de manipulation et renvoie à une mécanique de l'activité symbolique qui prive le destinataire de toute liberté et de toute parole propre (d'autant plus, bien entendu, s'il n'est pas éduqué). C'est cette idée, ainsi que le modèle de la communication comme transmission qui l'accompagne, que nous voulons questionner. Nous le ferons à partir d'un domaine empirique permettant de mettre en relief l'illusion qui caractérise l'idée d'une réussite de la communication : l'étude de systèmes de communication homme-animal.

\footnotetext{
' II est singulier qu'A. Culioli (1990) fasse usage de ce qu'il indique être son « aphorisme favori » au sujet d'une discussion sur l'activité signifiante développée dans une relation avec un animal (un chat).
} 


\section{Présentation de situations de communication}

Parmi les nombreux systèmes de communication homme-animal (chasse, cirque, animaux domestique ou de compagnie, animaux mangés, nuisibles, aidants ou chasseurs...), nous avons privilégié l'un des plus inhabituels : des rencontres enchantées entre des humains et des dauphins sauvages. Cellesci sont qualifiées d'enchantées car les personnes y font des expériences hors du commun : par exemple, elles entendent les dauphins leur parler ou font l'expérience de la dissolution des frontières du moi ou du « sentiment océanique » (Freud, 1929) qui caractérise les expériences mystiques². Le but n'est pas d'expliquer comment ces phénomènes surviennent, une ébauche en a été tentée ailleurs (Servais, 2005)3. En revanche, ces cas particuliers de communication entre humains et dauphins nous intéressent parce qu'ils posent problème à celui qui veut les décrire. Premièrement, il est impossible de supposer qu'ils reposent sur un code commun, contrairement à la communication avec les animaux domestiques ou de dressage. Ensuite, on se trouve en présence de deux créatures qui, par leurs dispositions biologiques et, pour ce qui concerne l'humain, sociales et culturelles, énactent ${ }^{4}$ (Valera, 1989) ou spécifient des mondes radicalement différents. Ceci pose la question du rapport entre communication et monde partagé. Enfin, leur analyse est compliquée par le fait que l'identification de ce qui est transmis (un signal ou un message) est ambiguë. Ces rencontres entre humains et dauphins ont encore d'autres propriétés dont celle de reposer largement sur l'émotion, de ne pas pouvoir être reliée - sinon de manière très délicate - à l'intentionnalité (du moins pour ce qui concerne l'animal) et de n'impliquer que de manière adjacente les dimensions sociales et institutionnelles de la communication. Pour toutes ces raisons, il s'agit de types de relations qui sont peu représentatifs des processus de communication faisant habituellement l'objet des recherches en sciences de l'information et de la communication (SIC). C'est là leur intérêt autant que leurs limites.

\footnotetext{
2 Expérience d'une non-dualité qui signe l'état fusionnel avec «l'Un ». La notion de « sentiment océanique » a été discutée par S. Freud en 1929 dans Le malaise dans la culture.

${ }^{3}$ Les témoignages qui seront utilisés ici ont été recueillis entre 1990 et 1996 dans le cadre d'une enquête ethnographique sur les types de rencontres entre humains et dauphins dans trois pays : Australie, France et Grande-Bretagne. Ils comportent des témoignages écrits et des témoignages recueillis oralement.

${ }^{4}$ Dans la définition du neurophysiologiste F.Varela (1988), l'énaction est la perception en tant qu'action. Par leurs actions les organismes, humains ou non, spécifient leurs mondes. Ceux-ci constituent alors leurs mondes propres. Dans la théorie de l'enaction, ce sont les actions concrètes des systèmes vivants qui instituent et spécifient ce à quoi ils sont sensibles dans l'environnement, leur monde ou niche écologique (Gérard et al, 2005). C'est ce phénomène de spécification d'un monde à travers l'action et la perception que désigne le terme d'enaction.
} 
Si nous avons choisi ces cas si particuliers, c'est que les difficultés méthodologiques qu'ils posent sont telles qu'elles obligent à envisager les processus de communication en dehors du modèle de la transmission, et à concevoir la communication au sein des interactions humainsdauphins comme étant structurée par un malentendu. C'est ce que nous tenterons de montrer dans la première partie, à partir de témoignages de rencontres enchantées. II est ensuite intéressant de comparer cette structure interactionnelle avec d'autres modèles de mécompréhension : la mésentente (Rancière, 1995) et le différend (Lyotard, 1983) 5 . Les notions de mésentente et de différend - telle qu'elles ont été développées et utilisées par leurs auteurs - engagent à développer la discussion dans de nouvelles directions : la politique où le peuple (au sens platonicien du terme) ne peut prendre la parole, faute de partager l'idiome du dominant (Rancières, 1995), d'une part, l'impossibilité de dire le génocide ou d'en transmettre simplement l'expérience (Lyotard, 1983, Coquio, 1999), d'autre part. En effet, e malentendu et le différend sont traités par ces deux auteurs en rapport avec la question du génocide ; la raison en est que, tout comme les situations de communication inter-espèce, ces situations demandent une appréhension différente de la relation entre code commun et communication. L'analyse de situations de communication inter-espèce nous sert donc à mettre en évidence des structures de communication qui, en fait, dépassent largement ce cadre restreint. En nous référant aux questionnements plus philosophiques de la communication que proposent les notions de mésentente et de différend, l'objectif est de montrer que le malentendu pose de facto une question politique, parce qu'il est en rapport avec l'hégémonie et le contrôle. Si ces situations, quoique très différentes par ailleurs, peuvent être rapprochées sur le plan de leur structure interactionnelle, il est envisageable de les rapprocher également sur le plan de l'analyse du contrôle et de la pathologie qui en est issue. Dans les interactions humains-dauphins, les tentatives pour contrôler, par la force, le comportement des animaux, apparaissent comme des tentatives de solution (Watzlawick et al, 1974), responsables de pathologies et sources de violence. Dans les situations humaines décrites, de même que dans les interactions quotidiennes qu'analysent les thérapeutes, des pathologies proviennent du refus d'accepter le fait que, d'une certaine manière, la communication est toujours structurée par un malentendu. II nous semble que cette perspective offre des pistes méthodologiques intéressantes; elle permet également de se désengager de la violence symbolique implicitement présente dans les modèles de la communication comme transmission d'un message.

5 || est remarquable que, lorsque le malendendu, le différend ou la mésentente sont évoqués dans la littérature, les animaux ne sont jamais bien loin. 
Dans ce cadre, notre but n'est évidemment pas de remplacer ou de nier la valeur d'analyses ou de modèles alternatifs : nous n'avons aucune prétention à l'hégémonie ou au fanatisme, qu'il soit disciplinaire ou méthodologique. D'autres analyses peuvent être faites à partir de nos témoignages, notamment dans le cadre d'une anthropologie de la communication (Hymes, 1967). Notre objectif est de développer un modèle possible pour l'analyse de situations de communication où, en l'absence d'un code partagé, les modèles reposant sur le code sont inopérants.

\section{Différend, mésentente et malentendu}

Avant d'aborder la définition du malentendu dans son rapport au différend et à la mésentente, revenons sur l'anecdote évoquée à propos d'Antoine Culioli. Le linguiste parle de malentendu à propos d'une question qui lui est posée au sujet de la définition de la linguistique. Antoine Culioli (1990: 39) ayant indiqué que l'activité de langage ne se limitait pas à « l'échange d'informations univoques, stabilisées et calibrées entre deux sujets qui seraient pré-ajustés pour que l'échange soit une réussite sans à-coups et sans faille », l'un de ses interlocuteurs en a inféré que l'interaction entre le propriétaire d'un chat et le chat pouvait relever d'une activité signifiante, et se demande, dès lors, si elle pourrait faire l'objet de la linguistique. Le linguiste corrige immédiatement en précisant qu'il ne confond pas activité signifiante et interaction. Au contraire de l'activité signifiante, l'interaction ne suppose pas que « les formes produites par l'énonciateur soient reconnues par le co-énonciateur comme étant produites pour être reconnues comme interprétables » (ibid. : 39). De la part des co-énonciateurs, elle ne suppose donc ni intentionnalité, ni accord sur ce qui constitue un signe. Le problème posé conduit directement au cœur du débat : comment rendre compte de l'interaction, voire d'une communication, sans supposer que le chat reconnait les signes comme devant être interprétés? C'est toute la question du code commun, ainsi que celle de la différence entre réaction et réponse, qui sont en jeu dans cette discussion autour de la définition de la linguistique. Selon Antoine Culioli, si cette dernière doit être en mesure de prendre en compte le malentendu (informations non univoques, pas de réussite sans faille de l'échange, etc.), il est nécessaire que celui-ci soit strictement limité par l'existence d'une intercompréhension possible et relative à l'identification des signes : le malentendu repose sur une entente possible (mais, nous verrons que, en définitive, cette position n'est pas tenable). Lever le malentendu signifie, alors, découvrir la règle commune sur laquelle s'élabore l'échange : dans le cas de cette discussion, il s'agit de l'accord sur ce qu'est un signe et sur ce qu'il n'est pas.

Cette question des limites et d'une règle commune nécessaires à l'intercompréhension est à l'origine du malentendu entre Antoine Culioli 
et son interlocuteur. Si les interlocuteurs ne sont pas pré-ajustés, ils sont néanmoins susceptibles de s'ajuster dans et par l'échange sur la base de la reconnaissance de ce qu'est un signe et de l'intention qui le qualifie. C'est bien ce qui caractérise une activité signifiante qu'Antoine Culioli n'est pas pressé de reconnaître au chat. Le malentendu vient de ce que le linguiste estime que la communication homme/animal est un malentendu (l'homme croit qu'il partage des règles avec le chat, alors qu'il ne peut y avoir de signe pour le chat), tandis que son interlocuteur la conçoit dans le cadre d'une entente ou d'un échange susceptibles de faire l'objet d'un accord. Mais alors, sur quelle règle commune reposerait le malentendu entre l'homme et son chat et, surtout, qui pourrait le lever? En l'occurrence, seul le savoir du linguiste peut lever le malentendu entre son interlocuteur et lui, c'est-à-dire un discours d'autorité édictant une norme à partir de laquelle la relation homme/animal peut être réinscrite dans une asymétrie salutaire pour la science linguistique.

Comme le montre cet exemple, la relation homme/animal pose au malentendu la question de la règle, singulièrement celle des règles de communication et, au-delà, de la norme. Si, dans ce cadre, le signe doit être redéfini par Antoine Culioli, c'est bien que ses limites doivent sans cesse - lorsqu'il s'agit des animaux - être réaffirmées ; ré-instituées, et cela parce que la relation homme/animal fait apparaitre leur « différance » (Derrida, 1968) 6 . En somme, s'agissant des animaux, ce que l'on découvre, lorsque l'on lève le malentendu, c'est la norme, imposée ici par la science linguistique. Le malentendu serait le lieu de l'institution de la norme et, par conséquent, du politique (Lyotard, 1983)7 II le serait non pas de manière contingente, mais nécessairement. C'est également en ce sens que nous voudrions l'interroger.

\section{Le malentendu et le différend}

Dans son ouvrage sur le malentendu, l'anthropologue Franco La Cecla (2002 : 23) insiste sur le fait qu'être d'accord ne signifie en aucun cas se comprendre. Plus précisément, le malentendu est « une divergence d'interprétation entre personnes qui croyaient se comprendre » (Robert, 1995). Catherine Coquio (1999:21-22) qui reprend cette définition du

\footnotetext{
${ }^{6}$ La différance, qui n'est « ni un mot, ni un concept », est proposée par J. Derrida en 1968 pour qualifier la structure du signe. On pourrait la définir brièvement comme l'impossibilité de la présence à soi du sens, ou comme l'altérité altérant l'identité, celle-ci ne pouvant dès lors se définir que dans le retard ou l'après-coup. Pour nous, il s'agit de suggérer que les limites du signe sont travaillées dans la relation à l'animal par ce qui les fait, en quelque sorte, différer d'elles-mêmes.

7 « La normative, qui est exclue de l'éthique, introduit à la politique » (Lyotard, I 983 : 207).
} 
Dictionnaire historique de la langue française Robert (1995), dans la longue introduction qu'elle consacre au malentendu, précise que « conflit qui s'ignore ou ignore ses raisons, [le malentendu] est un scénario d'échange désirant et raté qui, à la faveur d'un langage (ou d'un sentiment) commun, protège l'insu d'une divergence pour faire durer un accord trompeur, ou un désaccord opaque. D'où deux énoncés latents : mieux vaut bien s'entendre que se comprendre; mieux vaut se disputer que savoir pourquoi ». Dans cet ouvrage consacré à Parler des camps, penser les génocides, l'auteure estime que le débat sur les génocides fait l'objet d'un malentendu. Selon elle, la pensée même du génocide n'a lieu que dans une « culture policée du malentendu » (id.) qui protège le déni dont il fait l'objet et dont les victimes font les frais. Si la pensée du génocide fait l'objet d'un malentendu, c'est parce qu'elle s'articule autour de la notion de différend (et avec le différend tout ce qu'elle réunit sous la bannière d'une pensée nihiliste) qui, toujours selon elle, maintient dans et par les mots, une occultation esthétique du réel (valeur de l'indicible, de l'écriture du désastre) produisant l'autonomisation de la catastrophe dans un mythe, une esthétique, et empêchant que soient pris en compte les crimes réels. Cette pensée nihiliste, « axée sur un sens déréalisé, n’a pas pris acte de l'extermination réelle, qui est perte de vie et par là de sens » (ibid. : 26). Aussi le nihilisme est-il « l'allié objectif de la raison génocidaire. Qui marque un point décisif lorsque [...] l'intransmissible devient ultime contenu de transmission, sacré à ce titre tragique » (ibid. : 31). Le malentendu articulant le débat sur les génocides se caractérise par le fait qu'un langage commun occulte que l'on parle, en réalité, de choses différentes : événement exalté comme tel pour les uns, mort réelle pour d'autres. En d'autres termes, il y aurait malentendu parce que ceux qui parlent de différend se maintiennent dans la sphère symbolique (Catherine Coquio estime que, pour Jean-François Lyotard, le camp et l'extermination ne sont qu'un « objet culturel »), au pire, se paient de mots et négligent la réalité de la mort et de la souffrance. II convient de « retirer le «différend» post-génocidaire à sa fatalité linguistique, en révélant sa part idéologique : celle du malentendu mémoriel, qu'il faudrait hisser au statut de mésentente politique, au sens où en parle Jacques Rancière » écrit-elle (ibid. : 20-2I). Que signifie faire du différend, un malentendu, et lui donner, avec la dimension politique, le statut de mésentente?

Au cœur de cette polémique, figure la question d'une « transmission privée de tiers » (id.). Si, « comme l'a montré [ ... . Dionys Mascolo, l'identification au rescapé n'est au mieux qu'un émouvant malentendu, [...], l'événement génocidaire aurait-il donc pour particularité de ne livrer aucune vérité commune [...] ? » (ibid. : 29). De ce point de vue, cet événement relève bien alors d'un différend : « À la différence d'un litige, un différend serait un cas de conflit entre deux parties (au moins) qui ne pourrait pas être tranché équitablement, faute d'une règle de jugement applicable aux deux 
argumentations. Que l'une soit légitime n'impliquerait pas que l'autre ne le soit pas » (Lyotard, 1983 : 9). « Un cas de différend entre deux parties a lieu quand le "règlement" du conflit qui les oppose se fait dans l'idiome de l'une d'elles, alors que le tort dont l'autre souffre ne se signifie pas dans cet idiome » (id.). Dès lors, « le différend se signale par cette impossibilité de prouver. Celui qui porte la plainte est écouté, mais celui qui est victime, et qui est peut-être le même, est réduit au silence » (ibid. : 24-25). L'absence d'un idiome commun, d'une méta-règle, ne permet pas d'établir la réalité du tort : si celui-ci est effectif et si la victime peut en témoigner, ce n'est pas un tort qu'elle a subi mais seulement un dommage, et la victime n'est plus qu'un plaignant : telle est la structure du différend, qui est aussi celle de la double contrainte (Bateson, 1972). Aussi la transmission mémorielle des génocides a-t-elle également cette caractéristique : « Cette culture de la mémoire se constitue en système discursif sur une injonction paradoxale : devoir transmettre des événements sans pouvoir fixer leur sens aujourd'hui pour demain. Ce défaut de sens étant cela même qui les rend mémorables et litigieux » (Coquio, 1999 : 26).

À la différence toutefois de ce qu'affirme Catherine Coquio, cette impossibilité n'est, selon nous, nullement substantialisée ou esthétisée chez Jean-François Lyotard, puisqu'il ne cesse d'affirmer la nécessité de « phraser le différend ». «C'est l'enjeu d'une littérature, d'une philosophie et peut-être d'une politique de témoigner des différends en leur trouvant des idiomes [où] le vrai et le faux sont en jeu » (Lyotard, 1983 : 30 ; 35). Aucune extériorité n'est réellement possible pour qui cherche à penser l'événement génocidaire (Coquio, 1999 : 30). II convient d'aborder cet événement à la fois de l'intérieur et de l'extérieur (ibid. : 26). La tâche que se donne l'auteur, à travers une « saisie individuelle et collective des productions politiques de l'inhumain » (ibid. : 57) et la prise en compte du témoignage, en dehors du leurre de l'identification fusionnelle avec le rescapé, est bien de phraser le différend. Catherine Coquio appelle de ses vœux la possibilité de transmettre quelque chose sans que le sens en soit établi, de penser l'humanité contre l'unité, l'universalité, etc., c'est-àdire une pensée du malentendu. Pour cet auteure, c'est la reconnaissance du malentendu qui rend possible une pensée qui ne soit pas fondée sur l'unicité, sur l'universalité des normes. De ce fait, le malentendu serait le masque, la forme non reconnue du différend. Le différend génère le malentendu - autrement dit l'illusion de l'accord - lorsqu'il n'est pas reconnu comme tel, c'est-à-dire lorsque l'une des deux parties peut ou croit pouvoir imposer sa norme comme valable pour les deux parties ; reconnaitre l'existence d'un malentendu permettrait alors de faire émerger le différend et d'en rendre nécessaire l'explicitation, ainsi que l'acte de le phraser. C'est est une opération indispensable pour penser la pluralité des points de vue, prendre en compte la contingence de deux systèmes inconciliables, ainsi que pour identifier la domination. 
Si l'on applique ces réflexions au cas de la relation à l'animal, il apparaît que celle-ci est susceptible de relever non seulement d'un malentendu, mais encore d'un différend : impossibilité d'établir une règle commune aux deux idiomes ; absence d'universalité des normes ou d'unicité d'un système (de signes, de normes, etc.), mais reconnaissance de la contingence de deux systèmes ; absence de preuve de la réalité de l'événement - « le différend n'a pour objet aucune réalité établie » (Lyotard, 1983 : 50) ; nécessité de se situer à la fois à l'intérieur du système et hors de lui pour en rendre compte ; définition d'une impropriété comme propre. Tous ces éléments se trouvent dans la communication inter-espèces.

\section{Le malentendu et la mésentente}

Toutefois, la relation à l'animal relève encore de la mésentente. Définie comme « un type déterminé de situation de parole : celle où l'un des interlocuteurs entend et n'entend pas ce que dit l'autre » (Rancière, 1995 : 12), la mésentente se distingue du malentendu en ce qu'elle ne porte pas seulement sur les mots, mais aussi sur la situation de parole, et notamment sur le statut des interlocuteurs l'un par rapport à l'autre. Si le différend concerne lui aussi l'ensemble de la situation d'énonciation ${ }^{8}$, la mésentente a pour caractéristique de ne pas porter sur l'argumentation, mais sur l'argumentable, sur la présence ou non d'un objet commun aux interlocuteurs : «Elle concerne la présentation sensible de ce commun, la qualité même des interlocuteurs à le présenter » (Rancière, 1995 : 14). La mésentente renvoie à la possibilité ou non d'un « partage du sensible » avec comme situation extrême « celle où $X$ ne voit pas l'objet commun que lui présente $Y$ parce qu'il n'entend pas que les sons émis parY composent des mots et des agencements de mots semblables aux siens.

Comme on le verra, cette situation extrême concerne, au premier chef, la politique (Rancière, 1995 : 14). Par exemple, cette situation extrême est celle où le peuple, décrit comme gros animal par Platon, est considéré comme faisant usage de phonè et non de logos, exprimant la peine ou le plaisir, mais non le juste et l'injuste. La métaphore de l'animal, note Jacques Rancière, n'est pas une métaphore, mais « sert rigoureusement à rejeter du côté de l'animalité ces êtres parlants sans qualité qui introduisent le trouble dans le logos » (ibid. : 44). La mésentente concerne ce partage entre logos et phonè, où le logos est toujours aussi « le compte par lequel une émission sonore est entendue comme de la parole, apte à énoncer le

\footnotetext{
8 « Faire droit au différend, c'est instituer de nouveaux destinataires, de nouveaux destinateurs, de nouvelles significations, de nouveaux référents pour que le tort trouve à s'exprimer et que le plaignant cesse d'être une victime » (Lyotard, 1983 : 28).
} 
juste, alors qu'une autre est seulement perçue comme du bruit signalant plaisir ou douleur, consentement ou révolte $\rangle^{9}$ (id.).

Le politique survient lorsque se pose la question d'une scène commune où pourraient débattre de quelque chose ces deux parties dont l'une ne compte pas comme partie de la communauté. Dans cette situation paradoxale, il survient quand il faut faire entendre au peuple qu'il n'y entend rien ; il faut lui supposer le logos pour lui faire comprendre qu'il n'y prend pas part ${ }^{10}$. Le concept de mésentente renvoie lui aussi à la possibilité de phraser le différend, dans le sens où il décrit des phénomènes, où - à propos d'un objet sensible - apparaît la dissymétrie entre deux parties ; cette dissymétrie étant paradoxalement fondée sur une symétrie implicite, mais non reconnue. Jacques Rancière insiste d'ailleurs sur l'injonction paradoxale dans laquelle se déploie la mésentente, puisqu'il s'agit de communiquer à autrui qu'il est impossible de communiquer avec lui. Nous retrouvons la nécessité évoquée par Catherine Coquio de se situer à la fois à l'intérieur et à l'extérieur du système, de pratiquer ce que Jacques Derrida, à propos de la greffe, appelait une « double description ».

En résumé, nous devons reconnaître que le malentendu est susceptible de se résoudre par un accord possible sur la différence, mais cet accord engage nécessairement la référence à une norme, voire son imposition. Au contraire, le différend est insoluble dans le présent, mais appelle une nouvelle forme de description ou d'argumentation. La mésentente ajoute à cette structure la question d'un partage du sensible relatif à la reconnaissance que l'autre possède, lui aussi, le logos. La comparaison entre malentendu, différend et mésentente, ainsi que l'examen de leurs relations et composantes communes, donne un contexte à la situation d'interaction homme/animal ; ce contexte permet d'en saisir les enjeux: enjeux épistémologiques, puisqu'il est question d'une description fondée sur l'incomplétude et l'impropriété des événements décrits ; enjeux scientifiques, car nous pouvons nous demander quelle serait la discipline susceptible de fournir un tel point de vue ; enjeux politiques, puisque cette question renvoie, au fond, à la possibilité de rendre compte de l'altérité, de la pluralité, et de compter avec elle. Étant fondée sur une asymétrie et sur une altérité radicales, la relation homme-animal oblige à se donner des outils de description qui prennent en compte l'ensemble de ces questions.

\footnotetext{
9 Voir la position d'A. Culioli décrite supra.

${ }^{10}$ Bien entendu, on peut aussi exercer de la violence comme le font les régimes totalitaires. Il y a alors absence du politique au profit de la police.
} 


\title{
L'enchantement d'une communication sans mot
}

À la fin des années 90, une enquête ethnographique sur les rencontres entre humains et dauphins a permis de recueillir des données qui semblaient poser un défi à une description en termes de communication. II s'agissait de récits ou de témoignages d'expériences quasi mystiques, ou surnaturelles'", de rencontres avec des dauphins sauvages. Les humains en revenaient transformés. Au contact de l'animal, ils avaient expérimenté une communication directe et intime, immédiate et bouleversante, qui s'apparentait à une forme de révélation. Les dauphins leur avaient parlé ; ils avaient compris et appris sur eux-mêmes des choses qu'ils ne savaient pas auparavant, et nombreux étaient ceux qui, cherchant à expliquer ce qui leur était arrivé, ne trouvaient pas de mots assez forts. Parfois, la rencontre avait formé le point de départ d'un processus thérapeutique. Ces rencontres que nous avons appelées enchantées - sont caractérisées par la présence de deux éléments : d'une part, une communication vécue comme directe, de conscience à conscience, avec les animaux; d'autre part, la perte de conscience des limites du moi et l'expérience d'un lien avec l'ensemble de la création, qui donne leur caractère mystique aux rencontres. Toutes les rencontres avec des dauphins ne sont pas enchantées. Beaucoup sont banales et dépourvues du sentiment d'intercompréhension rapporté ici ; mais ce sont les rencontres enchantées qui nous intéressent. Par exemple, « c'est une personne transformée qui vous écrit » annonce Christina dans une lettre à la revue Dolphin ${ }^{12}$ :

\begin{abstract}
« Lorsque le choc de l'eau froide sur ma tête et mes mains diminua, j'ai pu flotter et regarder l'eau autour de moi, et Freddie 3 apparut juste à côté de moi. J'ai fait comme Gordon 14 m'avait dit, je lui ai caressé les flancs. L'amusement de Freddie lorsqu'il tourna ensuite sur son dos, tout à côté de moi, et resta pratiquement sans bouger, attendant plus de caresses ! [...]. Les dix minutes qui suivirent furent les plus merveilleuses de ma vie. Je lui grattais le dos, la tête et les mâchoires, et en retour, Freddie m'offrait de longues chevauchées - de la joie à l'état pur. C'était comme s'il répondait à mes sourires et mes rires en frappant ses nageoires sur l'eau [...]. Je pense aussi qu'il me comprenait, parce qu'une fois, il me fit faire une longue chevauchée loin du bateau, et en réalisant où j'étais, je lui ai demandé de me ramener au bateau, et il l'a fait ! ».
\end{abstract}

Dans ce récit, quoi que fasse le dauphin, Christina comprend. Elle n'a aucune peine à interpréter ce que fait Freddie comme des réponses à

\footnotetext{
"I Le terme de « surnaturel » renvoie aux expériences de télépathie.

${ }_{13}^{12}$ La Newsletter de l'association International Dolphin Watch.

13 Freddie est un dauphin solitaire qui a élu domicile au large de l'Irlande dans les années 90 ${ }_{4}$ et qui interagissait volontiers avec des humains.

${ }^{14}$ Gordon est un pêcheur du village qui offre son temps, son bateau et son expérience, à ceux qui veulent aller rencontrer Freddie.
} 
son propre comportement et à son égard: Freddie réagit à ses caresses en lui offrant son ventre clair, il répond à ses rires en frappant l'eau de ses nageoires; il offre une chevauchée contre des caresses et il la comprend: il fait demi-tour au moment où elle prend peur. C'est le cas de la majorité des récits enchantés : le dauphin émerveille les nageurs parce qu'il réagit de manière tellement sensée à leurs propres réactions ! En d'autres termes, le dauphin semble répondre ${ }^{15}$. La compréhension réciproque est bien l'une des marques de l'enchantement. Mais l'effet est encore plus fort quand l'animal donne l'impression d'agir intentionnellement :

«Quelques jours plus tard, nous fîmes l'expérience de notre plus forte communication avec les dauphins. Nageant parmi eux, nous avions repéré un très gros barracuda avec une sale gueule. De toute évidence, ils avaient conscience de notre crainte, car de temps à autre, ils éloignaient le barracuda. Le jour suivant, dans l'un des canots que nous traînions, nous trouvâmes, raide mort et desséché, le barracuda avec des marques de dents courbes sur son dos. Les dauphins semblaient l'avoir balancé là, tel un présent pendant la nuit, comme pour dire : "Hurumphff Barracuda... Pas de quoi avoir peur ! Tenez" » (Doak, 1981 : 240).

Parfois, les récits de rencontre avec les dauphins ont l'allure de transes hypnotiques légères et le thème de la communication par les esprits apparait :

« Face à l'océan, Doak entre dans une rêverie qui l'amène dans un état proche de la transe. Puis, soudainement et de manière tout à fait inattendue, "se produisit la rencontre homme-dauphin la plus élevée que j'aie jamais vécue. Quelque chose que je trouve très difficile à articuler autrement que par les faits à l'état pur. Durant au moins une heure, peut-être deux, six dauphins Stenella ont gambadé sous moi. II y avait une mère avec son petit et quatre autres. [...] Puis, commença une séance dans laquelle ils sautaient et retombaient en parfaite synchronie avec mes pensées, mes questions. Quelque chose que je n'arrive pas à croire, après coup, et dont je me souviens pourtant si clairement, comme les images arrêtées d'un film [...]. Dans le courant de l'après-midi, ils quittèrent tranquillement la baie, emportant tous les doutes que je pouvais avoir sur les liens entre esprits" » (Doak, | 98। : |87).

En effet, pour beaucoup de ceux qui l'ont expérimentée, la communication avec les dauphins est de l'ordre d'une communication par les consciences: « Le processus de communication [avec les dauphins] ne sera pas vocal, car nous en savons suffisamment maintenant pour reconnaitre que la communication chez les cétacés se fait par les esprits » a expliqué un orateur à une conférence (26-30/05/90) sur les baleines et les dauphins ${ }^{16}$. Les témoignages attestant des pouvoirs télépathiques du dauphin sont

\footnotetext{
15 S'agissant des animaux, la distinction entre réagir et répondre est au cœur du questionnement de J. Derrida (2006) dans L'Animal que donc je suis (voir par exemple p. 55 et toute la partie consacrée à J. Lacan, pp. 163-191).

${ }^{16} 2^{\text {nd }}$ International Dolphins \& Whales conference, Nambucca Heads, Australie, May 26-30, 1990.
} 
nombreux : « II semblait tout connaître de mes besoins, de mes désirs 》; « II avait perçu télépathiquement mes intentions »; « II lisait dans mes pensées ». I| arrive que la rencontre avec un dauphin sauvage affecte profondément l'être humain, comme c'est le cas de rencontres dites thérapeutiques :

\begin{abstract}
« À seize ans, explique Jemina, je suis devenue anorexique. Aujourd'hui, à 23 ans, je me bats encore pour m'extirper de ces difficultés, mais je suis convaincue que la raison pour laquelle je n'ai pas lâché prise ni rechuté, c'est que j'ai nagé avec un dauphin sauvage. Lors de notre première rencontre, Dorad m'approcha par derrière et en-dessous [...] Se tournant sur le côté pour me regarder avec un œil, il plongea délibérément son regard dans le mien. II n'y avait nulle part où se cacher. Cette créature sauvage ne regardait ni mon corps ni même mes expressions, mais droit dans la douleur de mon âme [...]. Et le regard d'un dauphin pourrait être la seule manière de calmer la douleur d'une terrible et très profonde blessure psychologique [...]. Dorad m'a appris comment regarder ma souffrance en face et l'affronter vraiment. J'ai fait l'expérience d'une confiance mutuelle et d'un amour inconditionnel, que peut-être seule une autre espèce extrêmement intelligente comme le dauphin peut offrir $\gg(1990)^{17}$.
\end{abstract}

Dans cet exemple, Jemina a fait une expérience correctrice qui s'accompagne d'émotion intense. En se regardant à travers les yeux du cétacé, elle découvre sur elle-même quelque chose qu'elle ignorait ou n'osait croire. La teneur de cette expérience est évidemment différente pour chacun, mais celle-ci est un aspect important de la communication enchantée, en particulier de la dimension de révélation de la rencontre. Quand le dauphin se met à « parler » à l'être humain, il lui parle, d'abord et avant tout, de lui-même ; et beaucoup des messages reçus par les humains peuvent être considérés comme une traduction approximative de ce qui est expérimenté dans la participation à l'interaction. Mais dans ce cas, quelle est la valeur de ces messages? II est difficile de croire qu'ils ont été effectivement envoyés par les dauphins.

\title{
Le vain motif de l'illusion anthropomorphique
}

« La littérature abonde de récits de dauphins "rieurs", "souriants", "jouettes", alors que ce que voyait l'observateur, c'étaient des claquements de mâchoires et des hochements de tête qui chez les dauphins signifient des intentions agressives » (Nollman, 1987, in : Pryor, Shallenberger, 1991: 163). Dans ce cas, les témoignages exaltés de rencontres avec un dauphin ne seraient que l'effet d'une profonde illusion anthropomorphique, d'une auto-suggestion comparable à celle dont est victime le propriétaire de chien lorsqu'il croit que son animal lui donne un baiser quand il lui

${ }^{17}$ Extrait de The Australian Dolphin \& Whale Journal (01/07/90). 
lèche le visage. C'est effectivement sur le terrain bien connu de l'illusion anthropomorphique que nous emmènent la plupart de ceux qui se sont intéressés à la communication inter-espèces (Hediger, 1980 ; Hediger, 198I ; Sebeok, Sebeok, 1980 ; Sebeok, Rosenthal, 1981; Despret, 2004). Tout comme il est difficile à l'éthologue de croire le chasseur qui lui explique comment l'animal chassé s'est offert à lui, il est impossible, à qui n'y croit pas, de souscrire à l'idée que les dauphins adressent des messages et communiquent télépathiquement avec les nageurs. À partir de là, il semble intéressant de commencer par distinguer, dans ces récits, ce qui se passe vraiment de ce que les nageurs croient, à séparer en somme ce qui relève de processus de communication véritables de ce qui relève de l'illusion ou de la croyance ; et ce, dans le respect d'une pertinence éthologique ${ }^{18}$. Toutefois, cette démarche de bon sens s'avère difficile à appliquer comme le montre le cas très élémentaire du regard :

\begin{abstract}
« Le matin, après mon arrivée à Dingle, j'endossai une combinaison de plongée, embarquai sur un bateau et dès que je vis l'aileron dorsal de Dorad, je sautai à l'eau. Presqu'immédiatement, il nagea près de moi et me gratifia d'un regard qui m'est resté depuis lors - un regard d'une grande intensité, de connaissance et d'acceptation [...]. C'est ce premier regard, d'une acceptation indicible, qui m'accompagnera pour toujours » écrit à Dolphin un Anglais reconnaissant.
\end{abstract}

« Les dauphins recherchent le contact visuel avec l'être humain » nous avait dit, sur le ton de la confidence cruciale, un énorme marin australien ; ce regard a déclenché de puissantes réactions émotionnelles : «Permettre à un dauphin de toucher votre cœur, c'est comme tomber amoureux » ; « J'ai perçu un sentiment de pur amour venant du dauphin... »; « J'étais ivre de joie, comme dans un rêve »; « J'ai posé ma main sur sa tête et j'ai quitté ce monde ». Rencontrer le regard d'un dauphin apparaît comme un échange interspécifique d'une rare richesse : « J'ai souvent lu des descriptions des sentiments que l'on a lorsqu'on regarde dans les yeux d'un dauphin, et c'est réellement indescriptible - comme regarder profondément dans l'âme de quelqu'un, une compréhension d'êtres d'intelligence égale » m'a dit un homme émerveillé. « Nous avons souvent regardé dans les yeux des dauphins, et la qualité de leur regard en retour ne ressemblait à celle d'aucun autre animal que nous ayons connu... », confirme, en 1990, la cétologue Ann Spurgon'19.

Il est aisé de se rendre compte que cet échange de regards modifie la scène de l'interaction et devient un élément essentiel de son contexte en y introduisant, précisément, l'intention. Dans les textes qu'il a consacrés

\footnotetext{
18 L'éthologie peut être définie comme l'étude du comportement (animal et humain) en tant qu'il est le produit de l'évolution (Renck, Servais, 2002).

${ }^{19}$ The Australian Dolphin \& Whale Journal (01/90).
} 
à la question de l'animal, Jacques Derrida (2006) fait l'hypothèse qu'il y aurait au fond, du point de vue de l'animal, deux grandes formes de traités sur l'animal : ceux qui sont signés par des gens qui ne se sont jamais vus regardés par un animal ou qui n'en ont tiré aucune conséquence, et ceux dont les auteurs ont pris en compte le fait « que ce qu'ils appellent "animal" pouvait les regarder et s'adresser à eux depuis là-bas, depuis une origine tout autre » (ibid. : 31 ). Selon Jacques Derrida (ibid. : 32), la dénégation de ce regard n'est pas une dénégation parmi d'autres, elle « institue le propre de l'homme, le rapport à soi d'une humanité d'abord soucieuse et jalouse de son propre ». II semble que c'est d'un tel franchissement de frontière qu'il s'agit ici et que les témoignages cités plus haut font état de la possibilité d'articuler - quoique de manière indécidable - le différend en un simple malentendu : tout se passe comme si l'on pouvait se comprendre, et cette possibilité, fût-elle fictive, a des effets bien réels sur l'interaction elle-même. En effet, la reconnaissance du dauphin comme sujet d'un monde et, au-delà, d'un savoir, d'un message, d'une intention bouleverse toutes les catégories des nageurs dès lors qu'ils reconnaissent que ce tout autre s'adresse à eux. Nous devons considérer qu'elle oblige également à reconsidérer les catégories de la communication et de sa réussite en général dès lors qu'une telle adresse est seulement possible. Mais comment la décrire?

Les nageurs parlent d'amour, d'émotion, de communication avec un être intelligent. Cependant, si l'on éprouve quelque difficulté à concevoir que les dauphins envoient délibérément de l'amour, il est tout aussi délicat de préciser en quoi consiste l'illusion. Qu'est-ce qui est faux exactement? L'attitude amicale des dauphins? La signification que donnent les nageurs aux émotions qu'ils ressentent? Du point de vue humain, celle-ci est pourtant éthologiquement valide ${ }^{20}$. Et si le sourire du dauphin est une illusion, quels sont les véritables signaux faisant fonctionner cette interaction? Quand on cherche à identifier précisément les illusions ou erreurs dont est victime le nageur et à les mettre en regard de faits éthologiques objectifs, on est conduit à confronter ce que croit le nageur à la signification réelle des signaux ou comportements du dauphin. Certes, le nageur se trompe quand il croit que le dauphin lui sourit, car ce qu'affiche le dauphin n'est qu'une particularité anatomique et non un véritable sourire. Bien sûr, le nageur a tort de croire que le dauphin lui envoie des messages : ce qu'il reçoit comme informations, sur lui-même par exemple, n’a probablement jamais été envoyé comme tel

20 Dans le système de communication, le regard mutuel signale et provoque un contact intime et un fort engagement émotionnel. Dans la vie sociale, le regard mutuel signale le début d'une interaction. Ici, cet élément est très important puisque, en signalant le début d'une interaction, il cadre la suite des comportements du dauphin comme autant de réponses aux comportements du nageur. 
par le dauphin. Mais il faudrait être dauphin soi-même (au moins) pour savoir ce que le petit cétacé envoie vraiment comme information. Et s'il est des cas où les humains prennent une menace (un claquement de mâchoires) pour du jeu, on n'a pas tout dit de l'interaction, une fois l'illusion dénoncée. Pas plus qu'on n'a expliqué l'enchantement, une fois considéré qu'il survient parce que les nageurs y croient comme nous l'avons souvent entendu dire par des collègues psychologues. Quand on oppose ainsi l'illusion à la vérité éthologique, l'enjeu est de condamner des gens et des pratiques plutôt que de comprendre comment fonctionnent des interactions. Cela ne permet en rien de comprendre ce qui se passe dans les rencontres, sauf à renvoyer toute l'explication sur le dos de l'anthropomorphisme, de l'irrationalité humaine ou de la croyance - ce qui n'explique rien. Au mieux, cela produit deux descriptions séparées, voire incommensurables : une description éthologique (réputée objective, scientifique, exacte) du comportement animal et une description ethnologique de pratiques et de croyances humaines où la question de la pertinence éthologique n'est pas posée. Mais aucune des deux n'envisage la rencontre comme communication et ne donne véritablement accès à l'interaction.

Ce que montre également cet exemple, c'est que, contrairement aux présupposés de l'anthropologie culturelle, un animal n'est pas un donné dépourvu de signification que des représentations purement humaines et culturelles viendraient saisir. Pour rendre compte de l'interface entre hommes et animaux, des anthropologues tentent aujourd'hui de faire place à l'animal en tant que sujet de son monde (Ingold, 1988 ; Reed, 1988 ; Brunois, 2005). Dans les rencontres enchantées, comme dans bien d'autres cas de communication inter-espèces, le recouvrement partiel des systèmes sémiotiques de l'animal et de l'humain (Bouissac, 1980) entraîne l'inadéquation des notions de message ou de signal. En effet, comme nous l'avons dit, la plupart des messages reçus n'ont jamais été envoyés et les signaux eux-mêmes sont instables, puisqu'il peut arriver que ce que le récepteur prend pour un signal n'en soit pas un du point de vue de l'émetteur. S'agissant de deux espèces différentes, les modalités sensorielles, les capacités perceptives et les processus cognitifs ne sont pas comparables. La communication ne peut pas être décrite comme transmission de messages. C'est d'ailleurs l'usage inconsidéré de ce type de modèle qui conduit les participants à croire que s'ils perçoivent des messages d'amour, c'est que le dauphin les leur a (intentionnellement) envoyés ; ce qui conduit à la profonde illusion dont nous avons parlé.

\section{Les critères d'une communication réussie}

Ainsi les auteurs de ces témoignages font-ils clairement état d'une expérience de communication qu'ils rattachent à cette image idéale de la communication comme transport, véhicule d'une représentation 
formée antérieurement, et au contenu lui-même idéalement préservé par le transport. Étant donné la dissymétrie qui existe de fait entre les deux systèmes sémiotiques, l'image est, dans ce cas, manifestement idéale voire fausse, bien que l'interaction produise des effets de présence (présence l'un à l'autre des interactants, présence à soi des individus), et un certain type d'effets intentionnels (conviction d'une intention accomplie permettant la poursuite de l'interaction) bien réels. Dès lors, doit-on considérer cette interaction comme un exemple de communication manquée, à la différence d'une communication réussie qui serait caractérisée par un transport de représentations fondé sur un accord (préalable ou consécutif) quant au sens ? La première question que posent ces témoignages est celle des critères d'une communication réussie. Clairement, parce qu'elle est fondée sur cette asymétrie des codes, la communication interspécifique pose la question de sa réussite d'une manière qui ne permet aucun choix définitif, aucune décision qui ne soit pas arbitraire. Ne pourrait-on pas considérer que c'est en réalité le cas, du moins en principe, pour toute forme de communication? En effet, que signifierait qu'une forme - ou un acte - de communication soit réussie ou manquée?

Pour cela, il faut aller plus loin et nous demander ce dont ces expériences peuvent témoigner, si on lève le malentendu sur lequel elles prennent corps, et ce que cela peut nous apprendre du signe ou de la communication en général. On a vu que, dans l'interaction, l'échange de regards joue un rôle prédominant car il permet de cadrer la relation comme réciproque. Si l'on se demande maintenant comment cela est possible (voir un animal s'adresser à soi), on peut apporter trois types de réponses. Au fond, le premier est celui adopté par Antoine Culioli (1990). Ainsi, dans le cas des interactions interspécifiques, les notions de message, de signe et d'intention, sont-ils instables et suspectes. Cependant, toute tentative pour discriminer, de manière simple, entre la réalité et l'illusion est vouée à l'échec : elle ne peut être utile qu'à une disqualification morale - et scientifique - de ces rêveurs qui se sont vus regardés par un animal, sans que pour autant l'on comprenne mieux ce qui se passe dans de tels échanges.

Une seconde réponse pourrait être celle qu'avance John R. Searle (199| : 228) avec la notion d'« intentionnalité collective ». Loin d'être l'apanage des humains, écrit-il, l'intentionnalité collective « semble plutôt être une forme biologiquement primitive de l'existence animale » (id.). L'intérêt de cette notion est qu'elle n'est pas réductible à des intentions individuelles auxquelles s'ajouteraient des croyances, car « les intentions en "Nous" sont primitives » (ibid. : 23I). Cette intentionnalité collective « présuppose, en arrière-plan, un sentiment pré-intentionnel de la communauté » (ibid. : 24I). Ainsi « le sentiment, biologiquement primitif, 
de l'autre personne comme un candidat à une intentionnalité partagée est une condition nécessaire de tout comportement collectif » (ibid. : 242). Outre la grande difficulté que rencontre l'auteur pour articuler l'acte collectif avec l'acte individuel, ainsi que le fait qu'il n'envisage pas de communautés interspécifiques, cette notion présente l'inconvénient d'offrir une explication faible à l'interaction : invoquer une prédisposition fût-elle biologique - des interactants à interagir ne peut constituer une explication, et encore moins si cette prédisposition doit elle-même s'accompagner d'une autre en arrière-plan. Enfin, préexistant aux actions, elle se présente aussi comme susceptible d'être pleinement remplie par les actes collectifs qui s'y inscrivent, et a un caractère clairement idéal, inentamé, puisque primitif, premier, naturel. On retombe dans le contexte d'une communication susceptible d'être réussie ${ }^{2 !}$.

Le dernier type de réponse pouvant être apporté à la question de savoir ce qui rend possible que les nageurs puissent ainsi se sentir interpellés par un animal qui s'adresse à eux, suppose de prendre ces témoignages au sérieux, tout en admettant qu'il soit impossible d'y distinguer, de manière définitive, le signe de l'illusion, la réalité éthologique du signe ou du signal, l'interprétation juste de la fausse croyance. En d'autres termes, il faut admettre la nature essentiellement fictive du signe ou, pour utiliser un terme plus adéquat, de la marque ${ }^{22}$. Si l'on se réfère à l'analyse que fait Jacques Derrida (1999) de l'itérabilité23 de la marque, on note que celle-ci me parvient de l'autre en tant qu'elle est déjà altérée par moi, par l'altérité que je constitue pour lui. De ce point de vue, la possibilité de la fiction ne dérive ni de la plénitude du réel, ni d'aucune littéralité, ni d'aucun langage ordinaire (ibid.) ; la possibilité de la fiction est toujours première, y compris dans l'expérience où, pour le dire vite, la représentation précède la présence. C'est exactement ce que montre le premier témoignage où la nageuse n'a cessé, pour raconter son aventure et en particulier faire

\footnotetext{
${ }^{21}$ Pour rappel, les critères de réussite d'un acte performatif sont pour J. Austin (1972) liés au contexte (exhaustivement définissable) dans lequel l'intention tient une place prépondérante. J. Derrida (1999) montre que ce critère de l'intention est indispensable à J. Austin dans la mesure où aucun critère interne à l'énoncé ne permet de définir le performatif, par rapport aux énoncés dits non sérieux, fictifs, etc. et donc d'en évaluer la réussite.

${ }^{22}$ Dans Limited Inc, J. Derrida (1999 : 128) revient sur la critique des concepts de représentation, d'expression, et de communication qui, « en appellent à quelque intention dissociable, intérieure et "derrière" I"'expression" », ainsi que sur sa suspicion à l'égard du signe, raison pour laquelle il substitue « marque » à « signe » et « effet intentionnel » à « intention ». II ajoute : « Je préfère toujours parler de l'itérabilité de la marque, au-delà de speech acts humains. Mes énoncés à se sujet [...] devraient valoir au-delà des marques et de la société dites humaines 》 (ibid. : 248).

${ }^{23}$ L'itérabilité est l'altération dans la répétition qui doit permettre de penser, à la fois, la règle et l'événement, le concept et la singularité (Derrida, 1999 : 120 ; 216).
} 
part des réponses du dauphin, d'user de ce « comme si » caractéristique : on ne sait si elle l'a vécu ainsi ou si elle a eu besoin de ces termes pour l'expliquer. C'est ce « comme si » qui supporte toute l'étendue du malentendu et vient masquer le différend, permettant que s'établisse et se poursuive l'interaction. C'est à cette condition de la possibilité essentielle de la fiction et de la dérive de la marque que l'on peut rendre compte de ce qu'une telle adresse du dauphin à l'homme est possible. L'itérabilité, qui re-divise autrement les oppositions telles que nature/ convention, rend compte de ce que toute marque est d'avance divisée par l'autre à qui elle s'adresse. Ainsi le risque de la fausseté (de l'échec) est-il nécessaire au fonctionnement même de la marque, pour autant que la communication ne soit pas purement répétitive, mais engage comme c'est très manifestement le cas ici - une forme de différence ou d'altérité. En d'autres termes, on pourra compliquer ou raffiner, comme on le voudra, le modèle télégraphique (par l'implication du contexte, de l'intention, etc.). La réussite de la communication ne peut être établie et le malentendu est une condition de l'entente ${ }^{24}$. Reste maintenant la question de la description de ces interactions. Si, dans une situation de différend, la réalité du référent n'est pas établie, ici, nous pouvons affirmer que la réalité même de l'interaction est problématique. Ce pourquoi les nageurs témoignent de leur expérience sans que le sens en soit fixé. À qui revient-il de le faire, et selon quelles modalités?

\section{Le malentendu comme double description}

L'alternative habituelle aux modèles télégraphiques de la communication réside dans les modèles orchestraux (Winkin, 1981). Selon Ray Birdwhistell (1970 : 230), la communication n'est pas un échange de cerveau à cerveau, mais peut se définir comme « la structure dynamique qui soutient l'ordre et la créativité dans l'interaction sociale » ou « un système complexe par lequel les membres d'une société s'inter-relient avec plus ou moins d'efficacité et de facilité » (ibid. : 12). Dans cette perspective, c'est ce système complexe dans son fonctionnement qu'il convient de décrire. Mais les modèles orchestraux ont un inconvénient majeur : bien qu'ils soient heuristiques sur un plan conceptuel, ils n'offrent guère de piste méthodologique permettant de les opérationnaliser dans un programme de recherche empirique. En effet, si chacun - qu'il le veuille ou non - participe à la communication, et si tout est communication (au moins potentiellement), on a besoin de critères pour choisir les unités

\footnotetext{
${ }^{24}$ Ce qui ne signifie pas, bien entendu, que l'entente soit impossible. Cela signifie seulement que le mal, l'échec, le « mis » sont toujours possible et que, comme tels, ils sont une condition de l'entente.
} 
comportementales à décrire. Dans ces conditions, il est intéressant de repartir de la notion d'information telle que la définit Gregory Bateson (1979 : 105) : une différence quelque part qui crée une différence ailleurs, et de considérer que le dauphin et l'humain sont chacun porteurs d'une infinité de différences. Parmi celles-ci, seule une faible partie sera perçue et fera réellement une différence. Une autre manière de présenter les choses est de considérer que le dauphin et l'humain sont chacun porteurs d'affordances ${ }^{25}$ qui seront énactées par le partenaire au cours de la rencontre, tel un processus de communication inter-espèces qui pourrait ressembler au schéma ci-dessous :

Figure I : Processus de communication inter-espèces.

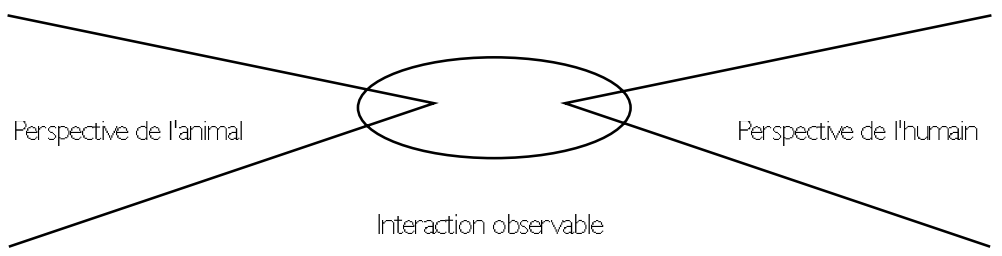

Au centre, l'ovale représente la partie visible de l'interaction. Cette partie est composée de séquences de comportements et, en principe, elle est objectivable. Elle fournit la ligne de base du déroulement de l'interaction : qui fait quoi, comment, à quel moment ? La partie de droite représente la perspective du participant humain sur cette interaction. Elle dépend de la position qu'il occupe dans l'interaction, de ses attentes, de son système sensoriel et perceptif, de ses apprentissages préalables, des habitudes culturellement acquises, des présupposés qui organisent sa rencontre avec l'animal, etc. Elle représente également l'humain et son monde tel qu'il l'énacte, ou le fait advenir par ses actions. De manière symétrique, la partie de gauche représente l'animal qui, lui aussi, énacte un monde propre en s'engageant dans une interaction avec un humain. Ce modèle repose sur une propriété fondamentale de l'interaction qu'a identifiée Gregory Bateson ( 1951 : 250) : « II n'y a pas de lecture objective d'une interaction, au-delà de la description des comportements comme s'ils étaient tous isolés les uns des autres »). Je peux décrire la rencontre entre un humain et un dauphin en décrivant ce que fait chacun : le dauphin plonge, l'humain met la tête dans l'eau, le dauphin se tourne sur le côté, l'humain ne bouge pas, etc. Mais, il manque ce qui relie ces comportements les uns aux autres. Or, l'unité élémentaire du lien social c'est « la réponse de A au comportement

\footnotetext{
${ }^{25}$ Dans la psychologie écologique de J. Gibson (1979), les affordances sont des opportunités pour l'action. Elles renvoient aux traits invariants de l'environnement que les animaux peuvent utiliser pour faciliter ou déterminer leurs propres actions.
} 
de $\mathrm{B}$ à son égard »(Bateson, 1935 : 221). La description objective d'une interaction réfère à une réalité qui n'existe nulle part ailleurs que dans le regard de l'observateur. C'est là tout le problème des analyses structurales de l'interaction. Quel est le statut exact des régularités objectives, des redondances ou des patterns qu'elles mettent en évidence?

Dans la pratique de la thérapie de couple, les thérapeutes notent régulièrement le caractère extrêmement répétitif des disputes qui suivent un schéma évident pour un observateur extérieur. Mais, pour comprendre la dynamique de l'interaction autrement qu'en termes de succession de comportements, nous avons besoin de savoir ce que le comportement $Y$ fait à Madame, et ce que la réponse de Madame fait à Monsieur, c'est-à-dire de décrire le point de vue de chacun sur l'interaction et le découpage particulier qu'il y opère. Comme le suggère notre schéma, décrire l'interaction revient à croiser les descriptions subjectives de chacun des participants. « On fait un grand pas si l'on commence à envisager deux parties d'une interaction comme deux yeux, chacun d'eux donnant une vision monoculaire de ce qui se passe, et les deux donnant ensemble la vision binoculaire de la profondeur: c'est cette double vision qui constitue la relation » (Bateson, 1979: 139). Pour rendre compte objectivement d'un système interactionnel comme l'enchantement, sans négliger la question du sens, il faut procéder à des doubles descriptions. On ne peut pas en rendre compte en postulant que seul l'humain construit une signification. En définissant l'interaction comme le produit de deux visions subjectives, ce modèle repose sur le postulat que chacun des partenaires construit son monde d'une manière ou d'une autre. En fait, il présuppose une définition phénoménologique du comportement humain et animal (von Uexküll, 1934) ; en cela, au vu des développements récents de l'éthologie cognitive (Gallo, 1988 ; Gallo et al, 1991 ; Delfour, Carlier, 2004), il est très contemporain.

Ce modèle possède également un certain nombre d'avantages dont le premier est probablement qu'il offre une réelle alternative au modèle inadéquat de la communication sociale qu'est le modèle télégraphique. Appliqué à la communication humaine, il considère comme inhérentes au processus de communication des propriétés que le modèle télégraphique considérait comme pathologiques ou anormales : par exemple, le simple fait que $A$ et $B$ ne peuvent concevoir un message $x$ de manière identique, parce qu'ils occupent des positions différentes dans une interaction. Une autre de ses particularités est de favoriser une vision interactionnelle de la communication. Parce qu'il oblige à recourir à des doubles descriptions, il met en évidence l'erreur qu'il y a à croire qu'une partie de l'interaction (l'un des protagonistes) peut contrôler l'ensemble. Comme l'ont amplement montré les praticiens de l'école de Palo Alto, la recherche du contrôle (sur la relation ou l'interaction, voire sur le partenaire) génère des pathologies. 
Considérer qu'une relation est toujours le produit de deux descriptions, c'est reconnaître que chacun - l'animal tout autant que l'être humain s'engage dans l'interaction pour y jouer sa propre partition (pour reprendre la métaphore orchestrale) et que, dans ces conditions, aucune des deux parties ne peut prétendre disposer de la bonne version de la relation ou du message. La perspective de l'humain n'est précisément que cela : la réalité qu'il construit par sa participation au sein d'une totalité qui le dépasse, la relation. Les systémiciens ont montré que, par définition, une partie ne peut contrôler le tout dont elle fait partie. Dans l'interaction, le contrôle est nécessairement une illusion. Bien entendu, un dresseur peut connaître suffisamment bien les règles du conditionnement opérant pour faire exécuter à son animal un saut périlleux. Temporairement, il peut croire qu'il maîtrise l'animal. Mais le bon dresseur sait qu'il est toujours à la merci d'un geste trop brusque, d'une femelle en chaleur, d'une humeur passagère, d'un environnement trop bruyant ou d'une punition en trop (Pryor, 1975 ; O'Barry, 1989 : 204-205). Dans les delphinariums européens, deux écoles de dresseurs s'opposent dans leur manière de gérer les comportements agressifs des mâles en captivité. La première cherche à les contrôler par la force, la punition, la privation de nourriture, l'isolement. Dans ces delphinariums, les dresseurs ont peur des dauphins et ils n'osent pas aller nager avec eux en dehors des spectacles. Dans l'autre tradition, les dresseurs essayent de faire avec les comportements agressifs et laissent les animaux régler leurs conflits en intervenant le moins possible. Dans ces delphinariums, les soigneurs peuvent se permettre de nager avec les dauphins en dehors du contexte de travail. Les premiers cherchent à contrôler et ils se retrouvent dans une relation incontrôlable au sens où ils ne peuvent pas faire confiance à leurs animaux; les seconds contrôlent beaucoup moins et peuvent faire confiance aux animaux... Visant la maîtrise des cétacés, les premiers s'engagent dans des escalades de violence parfois considérables ; les seconds ont la sagesse de reconnaitre qu'obtenir la soumission ou l'obéissance ne revient pas à avoir le contrôle. Dans les relations humaines, la conception d'une communication efficace, qui néglige totalement le fait que le récepteur, lui aussi, construit sa propre réalité, conduit à des pathologies de l'interaction.

Le modèle qu'on propose restitue au récepteur sa place et sa liberté d'interprétation. II possède un champ d'application très vaste, notamment parce que, ici, la question de l'intention à transmettre un message n'est pas pertinente. Si l'on accepte la prémisse du malentendu comme structure fondamentale de la communication inter-espèces (ce qui n'interdit pas que certaines significations soient partagées), les difficultés de description posées par nos rencontres entre humains et dauphins sont résolues. II devient vain de chercher à savoir si l'humain s'est trompé ou non, quel est le vrai sens d'un 
message, ou quand finit la vérité éthologique ${ }^{26}$ et quand débute l'illusion. La vérité éthologique n'est plus opposée à des croyances profanes et la question de savoir si le chasseur a tort ou raison, quand il pense que l'animal s'offre à lui, perd sa pertinence. On se donne également les moyens de décrire l'interaction en tant que système. Et à considérer l'interaction homme/animal comme un système, il est clair que l'enchantement ne se trouve ni dans le dauphin, ni dans l'être humain. II ne s'explique ni par des pouvoirs spéciaux du dauphin, ni par une illusion spéciale de l'humain (deux hypothèses dormitives), mais bien par le produit d'une interaction (de deux descriptions) qui, parmi plusieurs possibles, prend une configuration particulière. Tout en reposant sur une conception orchestrale de la communication, où chacun participe à la communication conçue comme un bain continuel de signaux circulant à de multiples niveaux, le modèle suggère des pistes méthodologiques. Par exemple, il serait possible d'identifier les affordances portées par l'animal et de les mettre en rapport avec les significations que l'humain leur donne, étant donné ses conceptions d'un animal, d'un dauphin, d'un message, de la communication, etc.

\section{La question de la méta-règle}

Si la vision objective de l'observateur ne correspond, en réalité, à aucune vision subjective, il s'ensuit qu'il n'y a aucune correspondance entre une méta-règle de régularité (observée par une tierce personne) et une méta-règle de prescription (que suivraient également les interactants) ou de traduction (permettant de traduire un idiome dans l'autre). Ainsi peuton constater que l'existence d'une méta-règle de régularité ne suppose absolument pas l'entente ou la reconnaissance d'une quelconque règle commune. II est important de reconnaître la contingence essentielle du rapport entre structure objective et vision subjective. Dès lors, c'est la notion de contexte qui devient problématique. D'une part, parce qu'il est impossible de le définir de manière exhaustive en adoptant une position de surplomb qui n'existe pas ; d'autre part, parce que l'interaction ellemême constitue une part de son contexte. On ne peut plus définir le contexte comme ce qui entoure l'interaction, mais il faut reconnaitre que, à la fois pour l'observateur et pour les interactants mais de manière différente, il en fait partie ${ }^{27}$ : « II n'y a que des contextes, sans aucun centre d'ancrage absolu » (Derrida, 1990 : 126). Toute description d'une interaction constitue, à la fois, une simple description et un geste discursif, se situe à un double niveau dénotatif et performatif qu'il faut assumer,

\footnotetext{
${ }_{27}^{26}$ C'est-à-dire une interprétation éthologique acceptée par les biologistes.

${ }^{27}$ On ne peut faire abstraction du contexte pour affiner l'analyse, note ). Derrida (1990: I 17) à propos des actes de langage, car « Le contexte est toujours déjà dans la place et non seulement autour d'elle ».
} 
notamment en termes de statut de la vérité. Cette double écriture, qui fait manquer la vérité, est la condition pour que quelque chose soit dit de l'interaction et de la communication qui s'approche de leur réalité. Définir la règle ou la prescription qui permet d'établir le contexte comme étant « le contexte », est, de ce point de vue, toujours un acte, une opération performative et non purement théorique. Et cet acte comporte toujours une part de violence, celle de l'institution de la règle ou de la loi. C'est pourquoi, comme le remarque Jacques Derrida (1990:25I), il y a toujours quelque chose de politique dans le projet même de tenter de fixer le contexte des énoncés :

« C'est inévitable, on ne peut rien faire, et surtout pas parler, sans déterminer (d'une manière qui n'est pas seulement théorique, mais pratique et performative) un contexte. Cette expérience est toujours politique parce qu'elle implique, dans cette détermination même, un certain type de rapport non "naturel" avec l'autre (et cela vaut aussi pour ce qu'on appelle les "animaux" car [...] ce que je dis implique une transformation assez profonde du concept de "politique" et de quelques autres pour qu'on puisse dire que l'homme n'est pas le seul animal politique) ».

\section{La domination éclipse le malentendu}

Dans le cas de communication inter-espèces, il est patent que, pour un dresseur, le contexte est constitué par une relation de domination, acceptée de part et d'autre, et qui explique que, dans le chef du dresseur, il ne puisse y avoir de dispute avec le dauphin. Dans ce cadre, l'animal ne peut répondre mais seulement réagir, et il n'y a aucune reconnaissance d'un quelconque malentendu. Au contraire, l'identification claire des règles de la relation suppose qu'il n'y a qu'une seule vision possible de l'interaction : l'animal obéit ou non. Si toute détermination du contexte implique un rapport de force, et si toute prise de parole implique, à son tour, la détermination d'un contexte, alors, il faut renoncer au primat normatif de l'activité communicationnelle que postulait jürgen Habermas (1992), même si celui-ci considérait que dans les actes de parole ouvertement stratégiques, les conditions de validité normative étaient remplacées par des conditions de sanction ${ }^{28}$. À l'inverse, pour les nageurs enchantés par la rencontre, la relation est cadrée par une forme de réciprocité qui rend l'animal capable de répondre et de s'adresser. Dans ce cas, la divisibilité du contexte, le fait qu'il n'entoure pas seulement l'interaction, mais aussi la constitue, apparaît de manière irréductible comme dans tous les cas où est prise en compte la possibilité même d'un malentendu; ; et le rôle du scientifique devient plus problématique,

${ }^{28}$ Pour une discussion de ces questions, voir K. O. Appel (|99| :26-32). 
car il implique - lui aussi - un choix d'ordre politique. On voit bien que c'est l'acceptation du malentendu qui, dans la mesure où elle entraîne l'abandon de toute tentative de contrôler l'autre et la reconnaissance de sa liberté de réponse, rend cette rencontre enchantée.

Cette reconnaissance d'une forme de responsabilité (au sens fort) de chacun, dans l'interaction, est, selon nous, nécessaire à toute communication non pathologique ; c'est pourquoi il est indispensable de considérer que le malentendu est une condition de l'entente et qu'il est une structure fondamentale de la communication. La volonté de supprimer le malentendu, de le résoudre en s'en rapportant à une règle, est toujours le moment où émerge la domination ; celle-ci se poursuivra tant que le dominé ne pourra se faire entendre dans l'idiome du dominant, prendre la parole depuis une place qui ne lui est pas reconnue, auquel cas l'interaction se transformera alors en conflit proprement politique. C'est la mésentente au sens où l'entend Jacques Rancière (1995), moment d'apparition sur une scène commune, de celui qui jusque là était sans part, hors communauté, hors scène. On peut considérer que l'une des marques et l'un des actes les plus forts de la domination consiste à refuser à l'autre l'accès au langage (au logos), à l'exclure de la possibilité même d'entendre la norme qui gouverne le code commun alors même que, comme le souligne Jacques Rancière (1995) ${ }^{29}$, il doit comprendre qu'il n'y comprend rien. Un code compris n'est en aucun cas, politiquement, un code commun. L'exemple de la communication enchantée montre que la possibilité de la fiction, au cœur de la marque, permet de phraser le différend en un simple malentendu. Sur le plan politique, Jacques Rancière (1995) montre qu'il est également nécessaire que celui qui est exclu de la communauté fasse comme si il en faisait partie en prenant la parole. Cet acte performatif est ce qu'il appelle apparition. C'est à cette condition de la fiction que peuvent être bouleversées les limites de la communauté. En d'autres termes, il est nécessaire d'accepter le malentendu pour qu'un différend puisse se phraser, pour que puisse apparaître sur la scène du politique toute forme d'altérité.

${ }^{29}$ Outre des textes proprement politiques, J. Rancière prend notamment pour exemple, plus proche de notre objet, l'enfant à qui l'on dit : «Tu as compris maintenant, hein ? ». Cette simple phrase, énoncée d'une certaine manière et dans certains contextes, suppose à l'enfant l'égalité (il doit être capable de comprendre), pour lui signifier l'inégalité (il doit se soumettre). 


\section{Conclusion}

La communication peut-elle réussir ? Peut-on dire qu'elle est réussie ? Choisir pour domaine empirique la relation homme/animal permet de mesurer toutes les implications d'une telle question, car il s'agit là d'un domaine où l'altérité ne peut être réduite. La comprendre et la décrire obligent à élaborer un modèle de communication fondé sur la pluralité qui est nécessairement une pensée politique. Chez Jacques Rancière (1995), c'est le partage du sensible, c'est-à-dire une valeur esthétique, qui en est le corrélat, comme d'ailleurs chez Emmanuel Kant où le sensus communis apparaît bien plutôt comme un dissensus communis ${ }^{30}$. De ce point de vue, un monde partagé n'est pas un monde commun ou que nous aurions en commun, mais cela signifie qu'il y a là un sensible qui nous tient ensemble, dans la mesure où il nous sépare, qui est notre ligne de partage et qui nous partage au sens où nous ne sommes pas identiques à nous-mêmes. Parler de monde partagé permet de penser notre différence à nous-mêmes; celle-ci est indispensable pour qu'un rapport à l'autre soit seulement possible. C'est pourquoi, penser la pluralité permet de ménager une possibilité de réponse qui ne soit pas une simple réaction; et le rapport à l'animal, parce qu'il interroge toutes ces questions, nous autorise à les réarticuler d'une manière plus fine. Lorsque Gregory Bateson (1935, 1972, 1979) montre qu'il n'y a pas de lecture objective d'une interaction, lorsque Jean-François Lyotard (1983), opposant le différend au consensus de Jürgen Habermas (1992), remet en question l'existence d'une méta-règle qui autoriserait in fine l'entente, lorsque Jacques Derrida (1968, 1990, 2006), par la déconstruction du signe linguistique et, au-delà de la notion de communication, lie - de manière essentielle - la question du sens à celle de l'autre et de l'altérité, chacun étaie cette idée qu'aucune partie d'un système ne peut prétendre exercer un contrôle unilatéral sur l'ensemble du système. De ce point de vue, il est assez clair aujourd'hui que la déconstruction du signe linguistique par Jacques Derrida est inséparable de la déconstruction de la frontière homme/animal' ${ }^{3 !}$.

Ce qu'a montré l'examen de la communication inter-espèces est que toute mesure, description, évaluation de la communication en termes de réussite, postule l'appropriation totale du sens de l'autre, son assimilation. C'est également ce que dit Franco La Cecla (2002 : 14) lorsqu'il définit le malentendu comme « une zone neutre, un terrain vague où l'identité,

\footnotetext{
${ }^{30}$ Voir aussi les travaux de H. Parret (1991).

${ }^{31}$ Sur l'animal comme trope majeur de la déconstruction, on peut lire le magnifique texte qu'É. de Fontenay consacre à J. Derrida dans Sans offenser le genre humain. Réflexions sur la cause animale (2008).
} 
ou mieux, les identités différentes et confrontées peuvent se positionner tout en restant séparées précisément grâce au malentendu. En ce sens, le malentendu peut défendre l'identité interne d'une personne ou d'une culture selon le schéma: «Tu ne me comprends pas? Cela vaut mieux; ainsi tu ne prétends pas que je passe dans ton espace et devienne comme toi ». Les enjeux politiques de ces conceptions respectives de la communication sont importants. En définitive, la question qu'ils posent est : que pouvons-nous accepter de l'autre ? Comment pouvons-nous penser le commun de telle sorte qu'il ne réduise pas l'altérité, de telle sorte qu'il n'y ait pas un sens en commun (Nancy, 1982, 1996), et que l'on soit ramené à la bonne traduction ou la bonne interprétation, mais que nous nous maintenions en rapport?

\section{Références}

Appel K.-O., 1991, « La fondation pragmatico-transcendantale de l'entente communicationnelle illimitée », pp. 15-33, in : Parret H., dir., La Communauté en paroles. Communication, consensus, ruptures, Liège, Mardaga.

Bateson G., 1935, La cérémonie du Naven. Les problèmes posés par la description sous trois rapports d'une tribu de Nouvelle-Guinée, trad. de l'anglais par J.-P. Latouche, N. Safouan, Paris, Éd. de Minuit, 1986.

- 1972, Vers une écologie de l'esprit, tome 2, trad. de l'américain par F. Drosso, L. Lot, E. Simion, Paris, Éd. Le Seuil, 1980.

— 1979, La nature et la pensée, trad. de l'anglais par A. Cardoën, M.-Cl. Chiarieri, J.-L. Giribone, Paris, Éd. Le Seuil, 1984.

Bateson G., Ruesch J., 195I, Communication et société, trad. de l'américain par G. Dupuis, Paris, Éd. Le Seuil, 1988.

Birdwhistell R.-L, 1970, Kinesics and Context. Essays on Body Motion Communication, Philadelphia, University of Pennsylvania Press.

Bouissac P., 198I, « In what sense is a circus animal performing ? 》, pp. 18-25, in: Sebeok T.-A., Rosenthal R., eds, The clever Hans phenomenon. Communication with Horses, Whales, Apes and People, Annals of the New York Academy of Science, 364.

Brunois F., 2005, « Pour une approche interactive des savoirs locaux : l'ethnoéthologie », Journal de la Société des Océanistes, 120-121, pp. 31 -40.

Coquio C., 1999, « Du malentendu », pp. 17-86, in : Coquio C., dir., Parler des camps, penser les génocides, Paris, A. Michel.

Culioli A., 1990, Pour une linguistique de l'énonciation, tome I, Paris, Ophrys.

Delfour F., Carlier P., 2004, « Expériences corporelles et reconnaissance de soi. L'exemple des mammifères marins », Hermès, 19 ( | -2), pp. 95-I 10.

Derrida J., 1968, Théorie d'ensemble, Paris, Éd. Le Seuil.

— 1990, Limited Inc., Paris, Galilée.

— 2006, L'Animal que donc je suis, Paris, Galilée.

Derrida J., Roudinesco É., 200 I, De quoi demain... Dialogue, Paris, Fayard/Galilée. 
Despret V., 2004, Hans, le cheval qui savait compter, Paris, Éd. Les Empêcheurs de penser en rond.

Dictionnaire historique de la langue française Robert, 1995, Paris, Dictionnaires Le Robert.

Doak W., 198I, Ambassadeur des dauphins, trad. de l'anglais par H. Verlomme, Br. Sifaoui, Paris, Lattès, 1993.

Dubois M.-.., 2005, « L'enracinement spatial des comportements chez les ongulés et les primates », pp. I39- I 54, in : Delfour F., Dubois M.-J., dirs, Autour de l'éthologie et de la cognition animale, Lyon, ARcI/Presses universitaires de Lyon.

Fontenay É. de, 2008, Sans offenser le genre humain, Réflexions sur la cause animale, Paris, A. Michel.

Freud S., 1929, Le malaise dans la culture, trad. de l'allemand par P. Cotet, R. Lainé, J. Stute-Cadiot, Paris, Presses universitaires de France, 1995.

Gallo A, 1988, « L'éthologie animale et le sujet », pp. 233-244, in : Gallo A., Ogé F., dirs, Homme, animal, société, Toulouse, Presses Institut Études Politiques.

Gallo A., Cuq C., Desporter, J.-P., 1991, « Épistémologie et éthologie », Revue internationale de systémique, 5 (4), pp. 47I-490.

Gérard J.-F., Dubois M.-J, Mechkour F., Bideau E., Maublanc M.-L., 2005, « Comportement et cognition animale : la perspective de l'énaction », pp. I55- | 82, in : Delfour F., Dubois M.-J., dirs, Autour de l'éthologie et de la cognition animale, Lyon, ARCI/Presses universitaires de Lyon.

Gibson J., 1979, The ecological approach to visual perception, Boston, HoughtonMifflin.

Habermas J., 1992, Droit et démocratie. Entre faits et normes, trad. de l'allemand par R. Rochlitz, Ch. Bouchindhomme, Paris, Gallimard, 1997.

Hediger H., 1980, « Do you speak Yerkish ? The newest colloquial language with chimpanzees », pp. 4| |-4|7, in : Sebeok T.-A., Umiker-Sebeok J., eds, Speaking of apes. A critical anthology of two-way communication with man, New York, Plenum Press.

- 1981, «The clever Hans phenomenon from an animal psychologist point of view », pp. I- 17, in : Sebeok T.-A., Umiker-Sebeok J., eds, Speaking of apes. A critical anthology of two-way communication with man, New York, Plenum Press.

Hymes D. 1967, «The anthropology of Communication », pp. I I-37, in : Dance F., ed., Human Communication Theory. Original Essays, New York, Holt, Rinehart and Winston.

Ingold T., ed., 1988, What is an animal ?, London, New York, Routledge.

La Cecla F., 2002, Le malentendu, Paris, Balland.

Lyotard J.-F., 1983, Le Différend, Paris, Éd. de Minuit.

Nancy J.-L., 1982, Le partage des voix, Paris, Galilée.

— 1996, Ettre singulier pluriel, Paris, Galilée.

O'Barry R., 1989, Behind the dolphin smile, Chapel Hill, Algonquin Books.

Parret H., 1991, « Communiquer par aisthèsis », pp. 183-200, in : Parret H., dir., La Communauté en paroles. Communication, consensus, ruptures, Liège, Mardaga. 
Pryor K., 1975, Lads before the wind, New York, Harper \& Row.

Pryor K., Shallenberger I., 1991, « Social structure in Spotted dolphins (Stenella attenuata) in the tuna purse seine fishery in the eastern tropical pacific », pp. 161-196, in : Pryor K., Norris K.S., eds, Dolphin societies. Discoveries and Puzzles, Berkeley, University of California Press.

Rancière J., 1995, La Mésentente, Paris, Galilée.

Reed E.-S., 1988, «The affordances of the animate environment : social science from the ecological point of view », pp. 1 10-126, in : Ingold T., ed., What is an animal ?, London and New York, Routledge.

Renck J.-L., Servais V., 2002, L'éthologie. Histoire naturelle du comportement, Paris, Éd. Le Seuil.

Searle J.-R., 199I, « L'intentionnalité collective », pp. 227-243, in : Parret H., dir., La Communauté en paroles. Communication, consensus, ruptures, Liège, Mardaga.

SebeokT-.A., Rosenthal R., eds, 1981, The clever Hans phenomenon : Communication with Horses, Whales, Apes and People, Annals of the New York Academy of Science, 364.

Sebeok T.-A., Umiker-Sebeok J., eds, 1980, Speaking of apes. A critical anthology of two-way communication with man, New York, Plenum Press.

Servais V., 2005, «Enchantging dolphins : an analysis of human-dolphin encounters », pp. 21 I-229, in : Knight J., ed., Animals in person, Oxford, Berg.

Uexküll von J., 1934, Mondes animaux et monde humain. Suivi de Théorie de la signification, trad. de l'allemand par Ph. Muller, Paris, Gonthier, 1965.

Varela F., 1988, Connaitre. Les sciences cognitives, tendances et perspectives, Paris, Éd. Le Seuil.

Watzlawick P.,Weakland J., Fisch R., 1974, Changements, paradoxes et psychothérapie, trad. de l'américain par P. Furlan, Paris, Éd. Le Seuil, 1975. 